\title{
Complex Materials for Molecular Spintronics Applications: Cobalt Bis(dioxolene) Valence Tautomers, from Molecules to Polymers
}

\author{
Arrigo Calzolari, ${ }^{* \dagger}$ Yifeng Chen ${ }^{\ddagger}$ Geoffrey F. Lewis, ${ }^{\S}$ Daniel B. Dougherty, ${ }^{\ddagger}$ David Shultz, ${ }^{\S}$ \\ and Marco Buongiorno Nardelli*,ll, \\ ${ }^{\dagger}$ CNR-NANO, Istituto Nanoscienze, Centro S3 I-41125 Modena, Italy \\ ${ }^{\ddagger}$ Department of Physics, North Carolina State University, Raleigh, North Carolina 27695, United States \\ ${ }^{\S}$ Department of Chemistry, North Carolina State University, Raleigh, North Carolina 27695, United States \\ "Department of Physics and Department of Chemistry, University of North Texas, Denton, Texas 76203, United States \\ ${ }^{\perp}$ CSMD, Oak Ridge National Laboratory, Oak Ridge, Tennessee 37831, United States
}

Supporting Information

ABSTRACT: Using first principles calculations, we predict a complex multifunctional behavior in cobalt bis(dioxolene) valence tautomeric compounds. Molecular spin-state switching is shown to dramatically alter electronic properties and corresponding transport properties. This spin state dependence has been demonstrated for technologically relevant coordination polymers of valence tautomers as well as for novel conjugated polymers with valence tautomeric functionalization. As a result, these materials are proposed as promising candidates for spintronic devices that can couple magnetic bistability with novel electrical and spin conduction properties. Our findings pave the way to the fundamental understanding and future design of active multifunctional organic materials for spintronics applications.

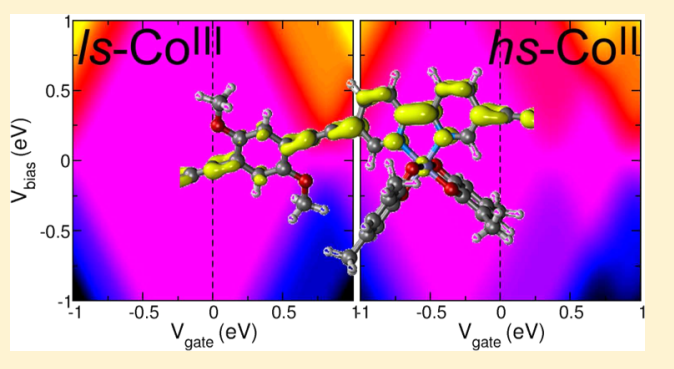

\section{INTRODUCTION}

Molecular spintronics seeks to exploit the electronic structural diversity of molecules to harness the spin of the electron in novel and improved computing and information storage applications. ${ }^{1-4}$ Significant material focus in this area has been placed on the class of conjugated organic semiconductors (e.g., rubrene and tris(hydroxyquinolato)-aluminum) that have found applications in organic optoelectronics devices. ${ }^{5}$ These materials, while often diamagnetic, have been reported to show dramatic spin-dependent charge transport effects including giant magnetoresistance (GMR) ${ }^{6,7}$ and tunneling magnetoresistance (TMR). ${ }^{8-10}$ However, recent years have seen net advances in the use of novel molecular materials synthesized for specific spin properties. This includes design and applications of single molecular magnets, which can have large spin quantum numbers and can be integrated into thin films ${ }^{11-13}$ suitable for device applications. ${ }^{14,15}$ In addition, the conjugated ferrimagnetic coordination polymer $\mathrm{V}(\mathrm{TCNE}) \mathrm{x}^{16}$ has very recently been employed as a thin film electrode in an all-organic TMR-based spin valve device. ${ }^{17}$

A molecular design motif with significant promise for spintronics is the spin crossover phenomenon ${ }^{18}$ in which a transition between high-spin and low-spin states of a coordination compound can be externally tuned by temperature, light, or applied pressure. This is very common in a number of $\mathrm{Fe}$ (II) compounds with nitrogen-containing ligands and has been suggested for many years as a route to molecular memory devices due to the prevalence of hysteretic effects in the spin transition. ${ }^{19}$ Recently, both experiment ${ }^{20}$ and theory ${ }^{21,22}$ have suggested spin state dependence of electrical transport in $\mathrm{Fe}(\mathrm{II})$ spin crossover compounds making them prime candidates for molecular spintronics.

A chemical elaboration of the spin crossover phenomenon can be established through the use of radical dioxolene ligands complexed to a cobalt coordination center in a class of compounds called valence tautomers (VTs). ${ }^{23,24}$ In valence tautomers, a high-spin to low-spin transition on the $\mathrm{Co}$ is accompanied by an intramolecular electron transfer from the Co to a redox active ligand.

This suggests a very dramatic coupling between electronic and magnetic degrees of freedom in the VT molecules that would be of great interest to spintronics. Moreover, recent calculations by Droghetti and Sanvito have predicted that the VT transition can be tuned by the Stark shift of molecular levels due to an applied electric field. ${ }^{25}$ However, to date almost no information exists about the connection between the VT transition and the transport of spin and charge in the VT material.

In this article, we identify a strong spin-state dependence of electrical and spin transport in valence tautomeric molecules and coordination polymers using first principles electronic structure calculations. Our results demonstrate that Co(dioxolene) valence tautomers indeed behave as multifunc-

Received: October 9, 2012

Published: October 16, 2012 
tional materials; i.e., they couple magnetic bistability with peculiar electrical and spin conduction properties that can be used in a variety of device architectures, ranging from molecular switches, sensors, and spin valves. This spin state dependence has been demonstrated for coordination polymers of valence tautomers as well as for a novel conjugated polymer with valence tautomeric functionalization.

\section{METHODOLOGY AND COMPUTATIONAL DETAILS}

Spin-unrestricted gas-phase geometry optimizations for both compounds $\mathbf{1}$ and $\mathbf{2}$ (see below) were performed at the density functional level of theory using the Quantum ESPRESSO software package. $^{26}$ All calculations employed the PBE exchange-correlation functional, ${ }^{27}$ a plane wave basis set with a kinetic energy cutoff of $30 \mathrm{Ry}$ (300 Ry) for the wave functions (charge), and ultrasoft pseudopotentials. ${ }^{28}$ The spin unrestricted calculations have been done using periodic supercells with a single $k$-point in the Brillouin zone $(\Gamma)$. Starting structures for $\mathbf{1}$ have been adapted from experimental crystallographic coordinates ${ }^{29}$ and fully relaxed. The starting geometry for polymer $\mathbf{2}$ was obtained from complexation of $\mathrm{Co}$ (dioxolene) units to a conjugated organic backbone, as suggested by the original synthesis experiments described in the Supporting Information.

While low-spin molecular configurations are obtained by spin unrestricted calculations, the high-spin states have been constructed by constraining the magnetization of the total system to the desired value $\left(\mu_{\mathrm{B}}=5\right)$ for both molecules. Since the singlet $l s$ and multiplet $h s$ configurations are the lowest energy states for the corresponding spin multiplicities, they are correctly calculated by DFT. ${ }^{30}$ An alternative approach, based on the consideration that the $l s \rightarrow h s$ transition is associated with an intramolecular charge transfer from the ligands to the Co atom, is to force the charge localization by imposing a penalty potential, like unscreened $\mathrm{LDA}+\mathrm{U}$, with an ad hoc $U$ parameter on $\mathrm{Co}_{3 d}$ states, chosen to reproduce the correct value of the magnetization and the associated Jahn-Teller distortion for compound $1 .^{29}$ However, given the large value of $U(\sim 8$ $\mathrm{eV}$ ), the resulting distribution of the Co states (on-site correlated) is fictitiously pushed down in energy far below the ligand state's levels (uncorrelated) and in clear contradiction with ligand field theory (see Figure S1 in the Supporting Information), confirming that the magnetic bistability of VT compounds is not directly related to Co electron correlation but rather to the specific interaction of Co with the attached noninnocent catecholate groups.

The intrinsic transport properties of the polymerized forms of the VT molecules have been computed in a lead/conductor/ lead geometry ( with lead $=$ conductor $=\mathrm{VT}$ polymer) using the fully first principles approach implemented in the WanT code. $^{31,32}$ The method combines an accurate description of the electronic ground state, provided by ab initio DFT calculations, with the Landauer approach to describe transport properties of extended systems. ${ }^{33,34}$ The connection is realized by transforming the Bloch orbitals into maximally localized Wannier functions. $^{35,36}$ This representation naturally introduces the ground-state electronic structure into the real-space Green function scheme, which is our tool for the evaluation of the Landauer quantum conductance. The method has been thoroughly applied to transition metal systems and is thus suitable for the present study. ${ }^{37,38}$

The absence of any type of self-interaction corrections (SIC) and the use of a functional that includes explicit exchange (such

as B3LYP) in our calculation prevents us from discriminating the magnetic order (ferro vs antiferro) within the single monomers and hence from evaluating the exchange coupling between Co and the ligands. ${ }^{25}$ However, this does not modify in any significant way the total magnetic configuration of the $l s$ / $h s$ states or the transport properties of these systems. At the same time, when the system is in the polymeric form, we can discriminate between intermonomer magnetic order (ferro- vs antiferro-magnetic) along the chain.

\section{RESULTS AND DISCUSSION}

1. Gas Phase Monomers. A. Valence Tautomeric Coordination Compounds. The cobalt bis(dioxolene) complex $\mathrm{Co}^{\mathrm{III}}(\mathrm{Cat})(\mathrm{SQ})(4-\mathrm{CNpy})_{2}$ (1) is composed of semiquinonate (SQ) and catecholate (Cat) forms of 3,5-dimetylbenzoquinone and two 4-cyanopyridine (4-CNpy) coligands. 1 (Figure 1) possesses a planar $\mathrm{Co}(\operatorname{diox})_{2}$ core with an effective
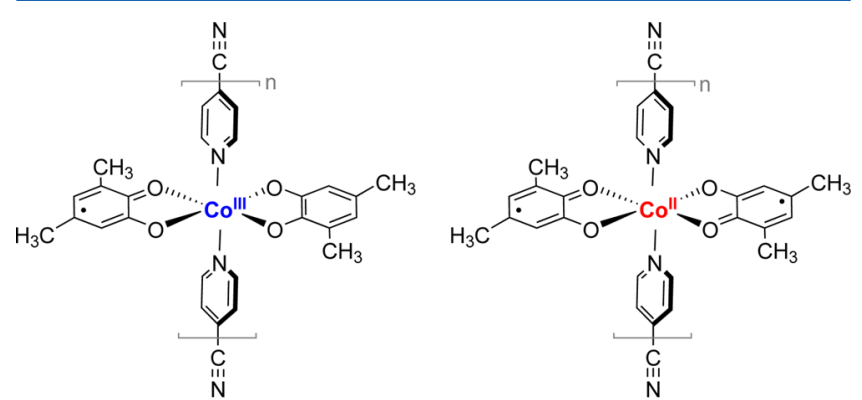

1

Figure 1. Structure of $1, l s-\mathrm{Co}{ }^{\mathrm{III}}(\mathrm{Cat})(\mathrm{SQ})(4-\mathrm{CNpy})_{2}(\mathrm{left})$ and $h s$ $\mathrm{Co}^{\mathrm{II}}(\mathrm{SQ})(\mathrm{SQ})(4-\mathrm{CNpy})_{2}$ (right). SQ = 3,5-dimetyl-o-semiquinone; Cat $=3,5$-dimetyl-catechol; 4-CNpy $=4$-cyanopyridine. Elements between square parentheses identify the repeated monomers in the corresponding polymeric structure.

octahedral symmetry. The ligand field splits the degeneracy of the atomic d-orbitals and results in three sets of frontier orbitals with well-defined symmetry: $t_{2 g}, e_{g}$, and $e_{g}^{*}$, where $t_{2 g}$ and $e_{g}^{*}$ originate from the usual ligand field spilt $\mathrm{d}$-orbitals and the $e_{g}$ set originates from ligand-derived $\pi$ orbitals (Figure 2). Depending on the ligand field strength, two valence tautomeric compounds may occur as a function of the molecular spin. In the strong coupling case, the Co ion is in the $\mathrm{Co}$ (III) oxidation state and forms the low-spin $(l s)$ state $l s-\mathrm{Co}^{\mathrm{III}}$ (Cat)(SQ)(4$\mathrm{CNpy})_{2}$; in the weak coupling case, the Co is in the Co(II) oxidation state and forms the high-spin (hs) state $h s$ $\mathrm{Co}^{\mathrm{II}}(\mathrm{SQ})(\mathrm{SQ})(4-\mathrm{CNpy})_{2}$ (Figure 1). The two configurations differ in charge distribution due to a multiple-step process involving also a Cat $\rightarrow \mathrm{Co}(\mathrm{III})$ electron transfer and a $l s \rightarrow h s$ spin crossover.

These two configurations are not only distinguishable by their different magnetic properties but also from their geometry. The Cat $\rightarrow \mathrm{Co}(\mathrm{III})$ electron transfer associated with the $l s \rightarrow h s$ transition is in fact accompanied by a JahnTeller distortion with a characteristic elongation of both the $\mathrm{Co}-(\mathrm{CNpy})$ and the $\mathrm{Co}-\mathrm{O}$ bond lengths of $\sim 10$ and $\sim 7 \%$, respectively: in our results, we go from 1.94 to $2.14 \AA$ for $\mathrm{Co}-$ $\mathrm{N}$ and from 1.89 to $2.04 \AA$ for $\mathrm{Co}-\mathrm{O}$, in excellent agreement with experiments. ${ }^{29}$

Our magnetic data confirm the picture of a $l s \mathrm{Co}^{\mathrm{III}}$ state with magnetization of $1 \mu_{\mathrm{B}}$ and a $h s \mathrm{Co}^{\mathrm{II}}$ state with magnetization of $5 \mu_{\mathrm{B}}$, where individual electronic levels are populated according 


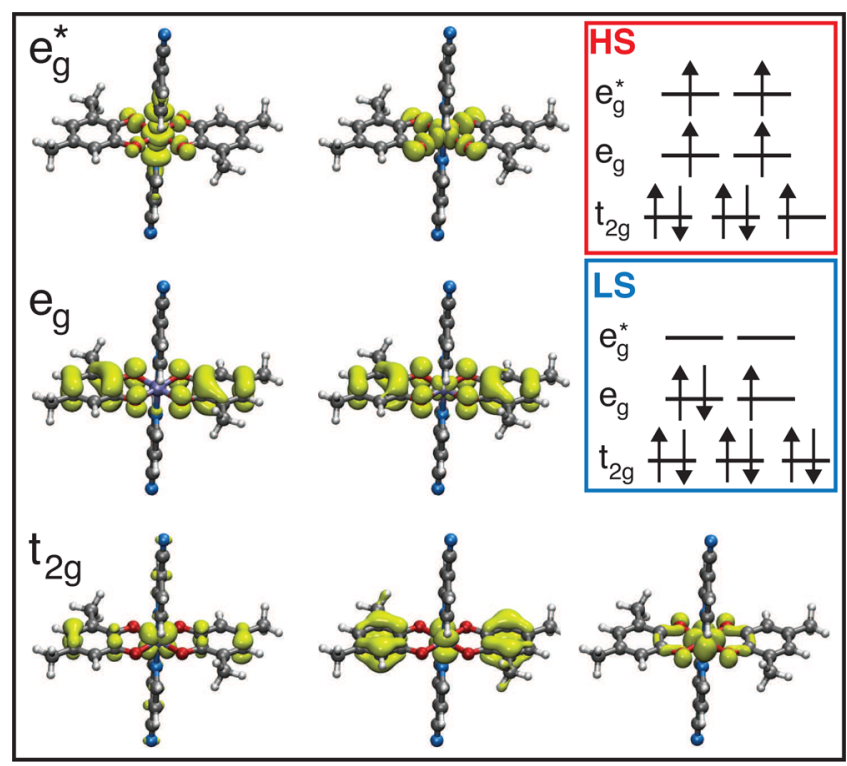

Figure 2. Selected wave functions $\left(|y|^{2}\right)$ corresponding to representative ligand field orbitals for molecule 1; (inset) spin configuration of the $l s$ and $h s$ states.

to the ligand field theory model as in the inset of Figure 2. For a thorough account of the full electronic structure of the systems, we have computed the total and Co-projected density of states for both $\alpha$ and $\beta$ spin channels. The results are displayed in Figure 3. Let us start from the $l s$ configuration: here, one can
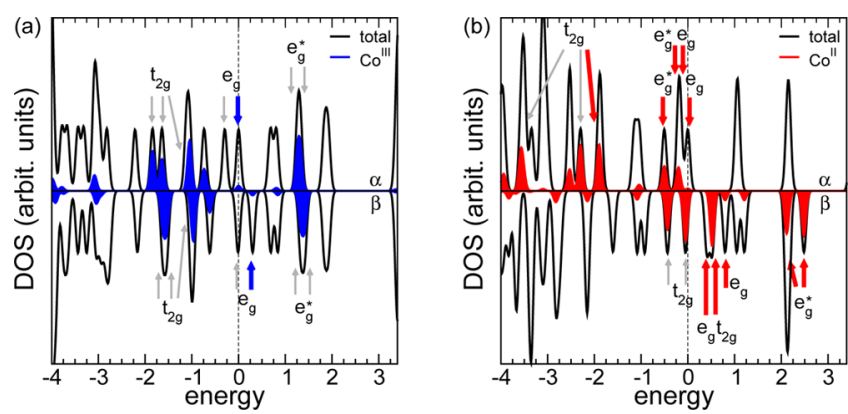

Figure 3. Total and Co-projected density of states for molecule $\mathbf{1}$ for both $\alpha$ (top) and $\beta$ (bottom) spin channels: (a) $l s$, (b) hs. Labels as in Figure 2. Gray arrows: double occupied states; blue (red) arrows: single occupied states for $l s(h s)$, respectively. The vertical dashed line marks the HOMO.

clearly recognize the class of high symmetry frontier orbitals, foreseen by ligand field theory. The Co-derived $t_{2 g}\left(e_{g}^{*}\right)$ orbitals are fully occupied (empty) in both spin components; on the other hand, the two ligand states $\left(e_{g}\right)$ are both occupied in the $\alpha$ channel, while only one is occupied in the $\beta$ one, giving rise to the expected $1 \mu_{\mathrm{B}}$ magnetization. In the $h s$ configuration, only two $t_{2 g}$ states are doubly occupied $(\alpha$ and $\beta)$, while the remaining others are singly occupied $(\alpha)$. This corresponds to five unpaired electrons, i.e., a total magnetization equal to $5 \mu_{\mathrm{B}}$. The single particle states corresponding to the above description are displayed in Figure 2 and in the absence of spin-orbit coupling, as in this case, their symmetry does not change from low- to high-spin, only their occupation. Finally, note that in our fully quantum mechanical calculations we are able to evaluate directly the lifting of the orbital degeneracies between the same couple of states with different spin.

B. Conjugated Monomers with Valence Tautomeric Functionalization. Organic materials for electronic applications often exploit conjugated polymers to allow efficient charge transport as well as facile solution phase spin-casting of device films. A possible approach to incorporating valence tautomerism into spintronic devices is to attach the tautomeric moiety directly to a conjugated backbone, to obtain a multifunctional material, which exhibits both switching magnetic properties (such as the cobalt-semiquinone VT complex 1) and electrical conductivity, with the final idea of exploiting the bistable magnetic property to control the extent of the latter.

The realization of conducting hybrid polymers with VT characteristics is in its infancy, and very few examples have been reported. ${ }^{39}$ Here, we propose a novel compound that could be considered for this purpose. The chemical structure is shown in Figure 4 and involves complexation of 2,2'-bipyridine segments directly to a $\mathrm{Co}(\text { diox })_{2}$ species. This compound has been recently synthesized in different configurations, with different decorating functional groups; the details of the chemical synthesis and the electro-optic and magnetic characterization are reported extensively in the Supporting Information.

As a starting point of our analysis, and similarly to the case of compound 1, we have studied the constituent monomer. In the conjugated cobalt bis(dioxolene) complex 2, the original planar symmetry of the $\mathrm{Co}(\text { diox })_{2}$ core of complex $\mathbf{1}$ is broken and now the system is characterized by a distorted octahedral symmetry. The ligand field still induces three sets of states that can be assigned as $t_{2 g}, e_{g}$, and $e_{g}^{*}$ (see Figure 5). However, we observe a much larger degree of hybridization between the Co and the ligand states than in the coordinated VT case. As for dyad 1, also here we observe a similar Jahn-Teller distortion associated with the $l s \rightarrow h s$ transition, with an elongation of the Co-N bonds of $\sim 10 \%$.

Our results show a net VT behavior, in agreement with the experimental magnetic susceptibility measurements reported in the Supporting Information. Our magnetic data confirm the picture of a $l s \mathrm{Co}^{\mathrm{III}}$ state with magnetization of $1 \mu_{\mathrm{B}}$ and a $h s$
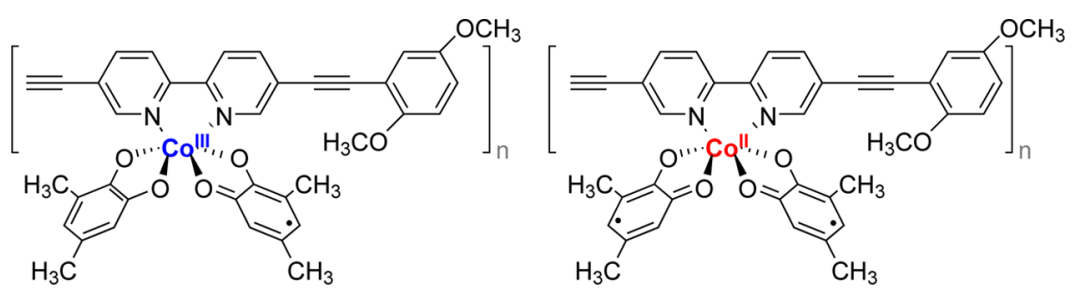

Figure 4. Structure of $2, l_{s}-\mathrm{Co}^{\mathrm{III}}(\mathrm{Cat})(\mathrm{SQ})(4-\mathrm{CNpy})_{2}$ (left) and $h s-\mathrm{Co}^{\mathrm{II}}(\mathrm{SQ})(\mathrm{SQ})(4-\mathrm{CNpy})_{2}$ (right). Elements between square parentheses identify the repeated monomers in the corresponding polymeric structure. 


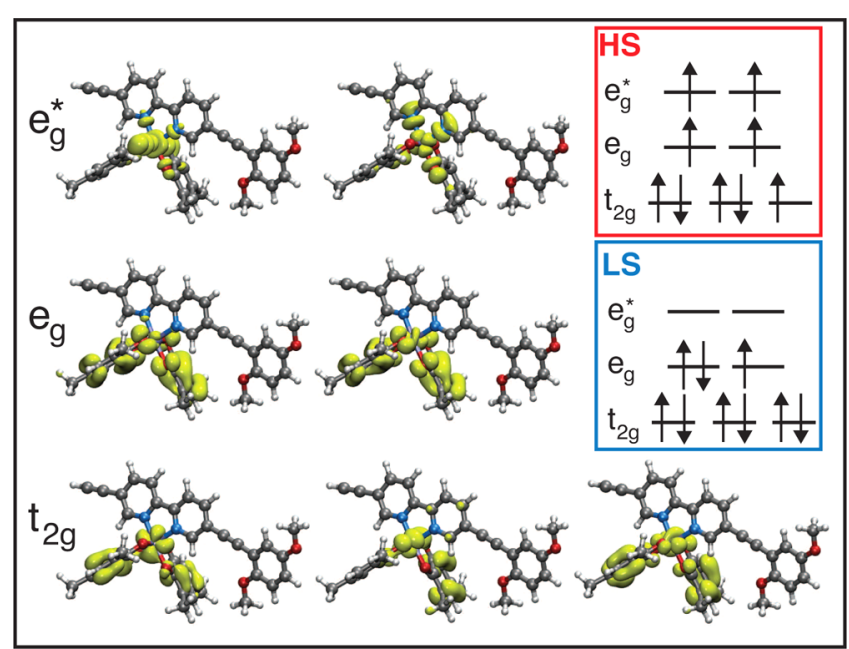

Figure 5. Selected wave functions $\left(|\psi|^{2}\right)$ corresponding to representative ligand field orbitals for molecule 2; (inset) spin configuration of the $h s$ and $l s$ states.

$\mathrm{Co}^{\mathrm{II}}$ state with magnetization of $5 \mu_{\mathrm{B}}$, where individual electronic levels are populated according to the ligand field theory model as in the inset of Figure 5. Also, in this case, we have computed the total and the Co-projected density of states for both $\alpha$ and $\beta$ spin channels. The results are displayed in Figure 6. The electronic structure of this dyad is very similar to
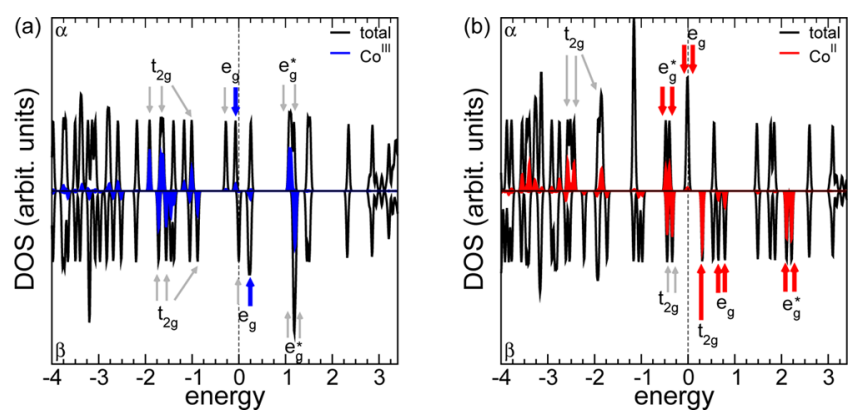

Figure 6. Total and Co-projected density of states for molecule 2 for both $\alpha$ (top) and $\beta$ (bottom) spin channels: (a) ls, (b) hs. Labels as in Figure 5. Gray arrows: double occupied states; blue (red) arrows: single occupied states for $l s(h s)$, respectively. The vertical dashed line marks the HOMO.

the previous case $\mathbf{1}$ for both $h s$ and $l s$ : the density of states follows the same ordering and occupations. The most striking difference between the two cases is in the symmetry of the single particle orbitals displayed in Figure 5. Although the character of the states (e.g., $t_{2 g}, e_{g}$, and $e_{g}^{*}$ ) is maintained, they lose the previous ideal octahedral symmetry because of the different structural motif and the steric hindrance in the molecular backbone. In particular, the conjugated character of the molecular backbone results in an enhanced hybridization between ligands and Co-derived states, and a larger delocalization of the electronic states along the molecular axis. With respect to the bare organic backbone (compound $\mathbf{2 b}$, Supporting Information), which has a DFT gap of $1.99 \mathrm{eV}$, the ligand field coupling with both catecholates and the cobalt generates a set of Co-ligand states (both occupied and virtual) that effectively reduce the pristine gap. This fact plays a fundamental role in the definition of the transport properties of these systems (see below).
2. Extended Polymers. For the utilization of these molecular systems in spintronic applications, one of the possibilities is to assemble the molecular units in polymeric structures which, while preserving the valence tautomeric bistable character, enhance the transport properties and allow for easier integration in device architectures. Ongoing experimental efforts are focusing on the synthesis and electrical characterization of quasi-1D polymeric chains self-assembled from molecular precursors in fibers (see the Supporting Information).

The direct measurement of the electron transport through single or few polymer chains is a tremendous task due to the difficulty in the extraction of the wires from the native fibers and in the formation of contacts at the nanoscale. ${ }^{40}$ To our knowledge, no measurements yet exist for the electronic (or spin) transport properties of isolated VT polymers to date.

In what follows, we discuss the electronic and transport properties of the polymeric structure obtained from our first principles methodology for coordinated (conjugated) compound 1 (2), respectively.

A. Valence Tautomeric Coordination Polymers. Coordinated polymer $\mathbf{1}$ is obtained from molecular monomer $\mathbf{1}$, upon removal of two $\mathrm{C} \equiv \mathrm{N}$ terminals (Figure 1 ). In our simulation, we included two monomer units in a supercell geometry and let the system relax for both $l s$ and $h s$ spin configurations. In both cases, the resulting polymer has a principal axis stemming from the sequence of a Co ion every two CNpy units, while the dioxolene ligands remain perpendicular to the polymer axis.

The vertical periodic pitch $\left(a_{0}=21.86 \AA\right)$ for the $l s$-Co ${ }^{\text {III }}$ polymer was extrapolated directly from the corresponding molecular structure. Peierls-like intermonomer distortions are not observed. In the case of the $h s-\mathrm{Co}^{\mathrm{II}}$ configuration, in order to take into account the Jahn-Teller distortion associated with the magnetic transition, ${ }^{41}$ the overall length of the polymer $\left(a_{0}\right)$ was optimized by varying the longitudinal size of the supercell and keeping the two transversal dimensions fixed, in order to maintain the lateral separation between adjacent replicas. For this optimization, we did total-energy and force calculations at a number of selected lattice parameters (i.e., varying the $\mathrm{Co}-\mathrm{N}$ distances) and relaxing all the atomic coordinates until the forces on all atoms were smaller than $30 \mathrm{meV} / \AA$ for each selected lattice constant. The results (see Figure S2, Supporting Information) show one local and one global minima (at 22.57 $\AA$ ). The latter value was assumed as the equilibrium polymer length. This corresponds to a $\mathrm{Co}-\mathrm{N}$ distance equal to $2.10 \AA$, i.e., to an elongation of $9 \%$ with respect to the $l s-\mathrm{Co}^{\mathrm{III}}$ case.

The electronic structures of the two polymers closely follow the corresponding molecular configurations. In particular, the molecular-orbital character of the electronic states (e.g., $t_{2 g}, e_{g}$, and $\left.e_{g}^{*}\right)$, their energy distribution, and the magnetization per monomer remain similar to the molecular cases. The results are reported for completeness in the Supporting Information, Figure S3.

Following the procedure outlined above, we calculated the coherent transport (i.e., no dissipative scattering) through the polymer within ideal (scatter-less) contacts; see the Supporting Information for further details. Generally, the details of the conductor/lead interfaces as well as spatial orientation of the polymer within the device may certainly change the transport properties of the device. In the absence of any direct experimental results, we here focus on the intrinsic conduction properties of the copolymers that are the theoretical prerequisite for any two-terminal device simulation. 

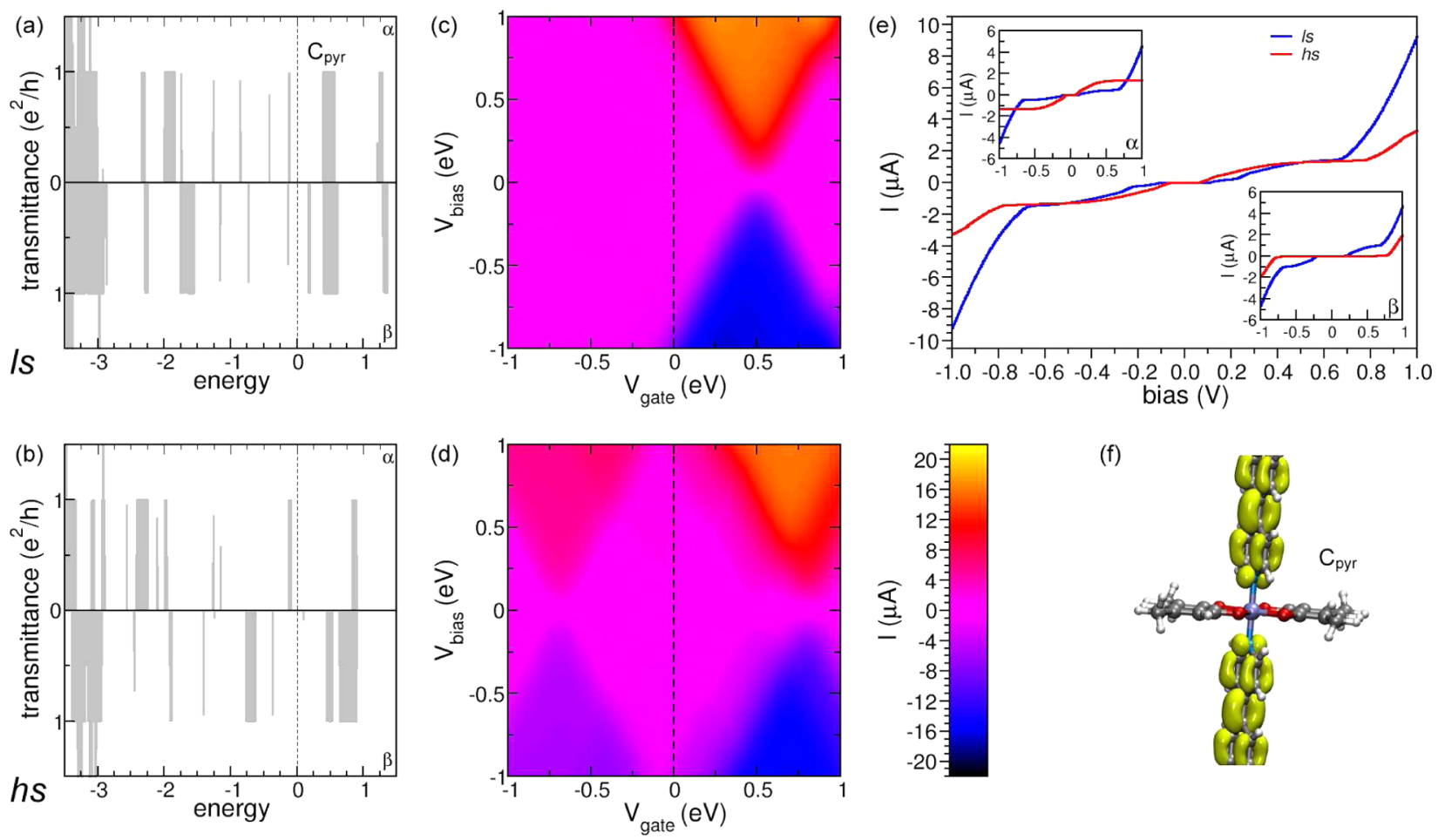

(f)

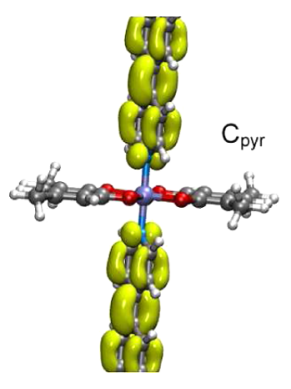

Figure 7. (a) Spin resolved transmittance spectra $\left(\mathcal{T}_{\text {el }}\right)$ for polymer 1 in the $l s$ configuration; (b) same as part a but for the $h s$ configuration; $(\mathrm{c})$ current as a function of both bias and gate voltage for $l s$; (d) same as part c but for $h s$; (e) current vs bias voltage at $V_{\mathrm{g}}=0 \mathrm{eV}$, insets: spin resolved currents for the $\alpha$ and $\beta$ channels; (f) transport eigenchannel for one representative state labeled $\mathrm{C}_{\mathrm{pyr}}$ in part a.

In periodic systems such as the polymers, there is a one-toone correspondence between the transmittance and the electronic (band) structure. In fact, in the absence of external leads, at a given value of energy $E$, the quantum transmittance is a constant proportional to the number of transmitting channels available for charge mobility (see the Supporting Information), which are equal (in a periodic system) to the number of conducting bands at the same energy. However, not all the states (bands) contribute to transport (see Figure S6 in the Supporting Information). For instance, states that are strongly localized on the dioxolene ligands (i.e., perpendicular to the transport (axial) direction) do not have any component in the transmittance spectrum; thus, a full calculation of the transport properties within a Green function approach is mandatory.

The transport results for polymer $\mathbf{1}$ are summarized in Figure 7. Let us first analyze the behavior of the transmittance spectra $\left(\mathcal{T}_{\text {el }}\right)$ for both the $l s$ and $h s$ configurations and both spin channels (Figure 7a,b). In a molecular system, the transport eigenchannels (i.e., the eigenvectors of transmission function ${ }^{42}$ ) coincide almost completely with the extended molecular orbitals of the system. The analysis of these orbitals gives us direct information on the path of the spin current in real space. From our results, it is clear that the character (symmetry) of the states that contribute most to transport do not change from $l s$ and $h s$ in both spin channels but their position in energy is modified in the different magnetic configurations. In general, states that contribute have either a component on the CNpy (delocalized $\pi$ orbitals) or are derived from the Co metal complex with a strong axial component. Starting from the calculated transmittance spectra, we have computed the current in the linear regime following the procedure in refs 31 and 32:

$$
I(V)=\int_{-\infty}^{+\infty} \mathcal{T}_{\text {el }}\left[f\left(E-\mu_{\mathrm{L}}\right)-f\left(E-\mu_{\mathrm{R}}\right)\right] \mathrm{d} E
$$

where $f$ is the Fermi distribution function, $\mu_{\mathrm{L}, \mathrm{R}}$ are the chemical potentials of the (left, right) leads, and $\mu_{\mathrm{L}}-\mu_{\mathrm{R}}=e \cdot V$ is the applied bias. Depending on the applied bias or on the position of the Fermi level, the contributions of the various conductance peaks change and so does the integrated current. Experimentally, the Fermi energy can be moved by the application of a gate voltage. Note that, in our linear response approach, we do not take into account any capacitive effects that might appear due to short-range electron-electron correlations: we only consider currents due to electron tunneling between bandlike states; i.e., we are not in a Coulomb blockade regime.

The total currents $\left(I_{\alpha}+I_{\beta}\right)$ as a function of the applied bias and gate potentials are displayed in Figure $7 \mathrm{c}$ and $\mathrm{d}$ for the $l \mathrm{~s}$ and $h s$ configurations, respectively. It is clear that the behavior of the system is vastly different in the two cases: the diamondshaped current distributions in panels $\mathrm{c}$ and $\mathrm{d}$ are indicative of a bistable on/off current behavior as a function of the gate voltage. In the $l s$ configuration, the current is maximized at $V_{\mathrm{g}}=$ $0.5 \mathrm{eV}$, in correspondence with a $\pi$-like conjugated state (labeled $\mathrm{C}_{\text {pyr }}$ in panel a and displayed in panel $\mathrm{f}$ ), while it remains zero for the majority of the bias range. On the contrary, in the $h s$ configuration, the current distribution is more symmetric with the gate and shows a wider range of small but not negligible current (see, for instance, for $V_{\mathrm{g}}$ between -0.5 and $0.5 \mathrm{eV}$ ). By forcing the system at a negative gate voltage, one can effectively design a switch (or sensor) where the current can be turned on-off by the modification of the spin state of the polymer, for instance, by photoirradiation. Alternatively, one can use this property in molecular sensors to detect the magnetic state of the polymer by a simple current measurement without any explicit magnetic probe.

It is important to note that, at any gate potential not coinciding with a molecular orbital, the current shows an 

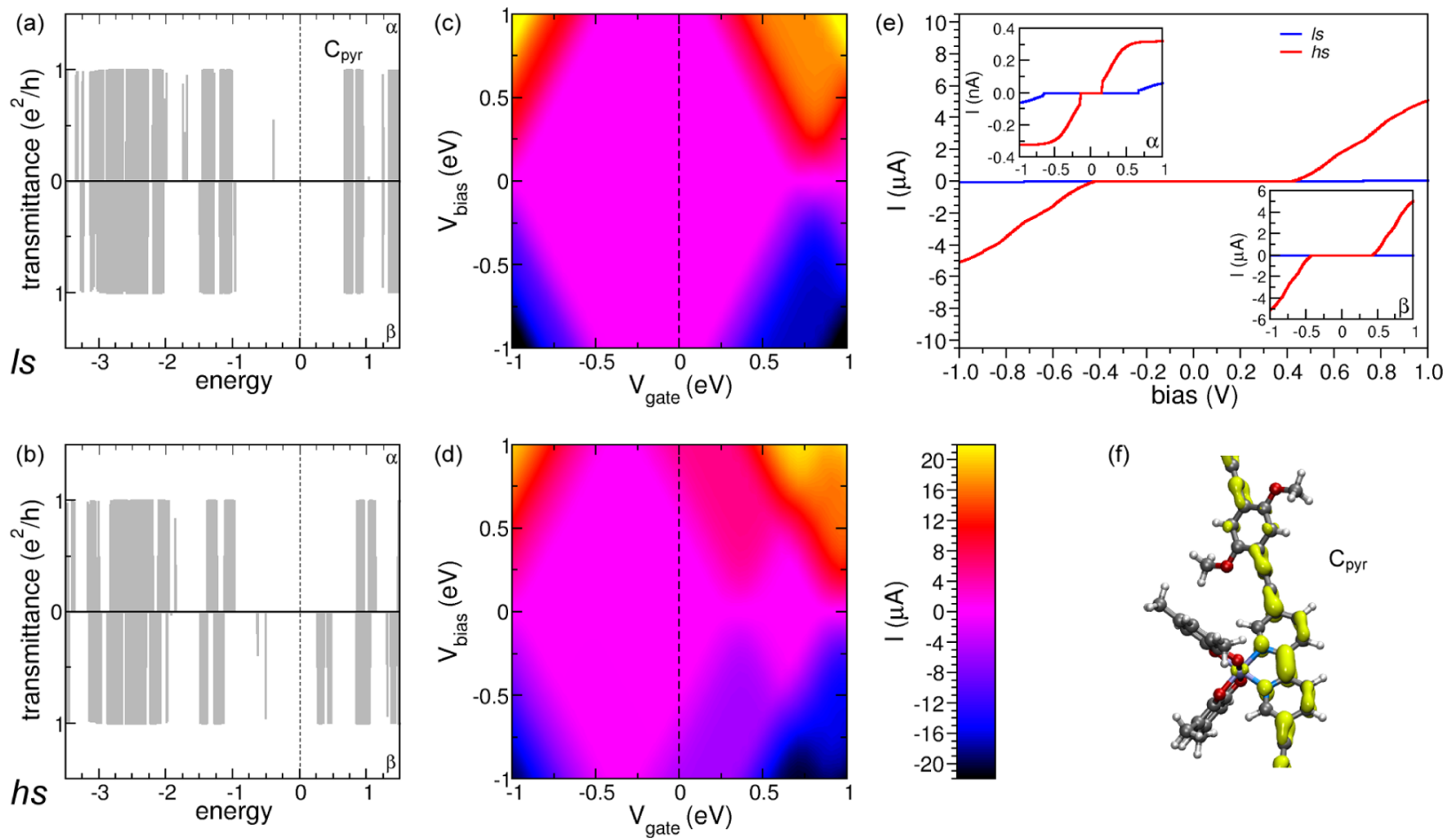

Figure 8. (a) Spin resolved transmittance spectra $\left(\mathcal{T}_{\text {el }}\right)$ for polymer 2 in the $l s$ configuration; (b) same as part a but for the $h s$ configuration; $(c)$ current as a function of both bias and gate voltage for $l s$; (d) same as part c for $h s$; (e) current vs bias voltage at $V_{\mathrm{g}}=0 \mathrm{eV}$, insets: spin resolved currents for the $\alpha$ and $\beta$ channels; (f) transport eigenchannel for one representative state labeled $\mathrm{C}_{\mathrm{pyr}}$ in part a.

energy gap. This is a consequence of the fact that the polymer 1 is indeed a semiconductor. The difference in currents between the two magnetic states can be easily interpreted in terms of the individual $\alpha$ and $\beta$ spin channels. In Figure 7e, we display the two spin components of the current for the $l s$ and $h s$ configurations at zero gate voltage for reference. Although the total currents are very similar, it is clear that, in the $l s$ state, the $\beta$ channel is dominant in a wide range of bias voltages, while the opposite is true for the $h$ s case.

B. Valence Tautomeric Pendants on a Conjugated Polymer. At variance with $\mathbf{1}$, the polymerized form of the conjugated VT molecule 2 loses the linearity of the coordination polymer with a $\mathrm{CNpy}-\mathrm{CNpy}$ angle of $176^{\circ}$. To allow periodicity in the axial direction, we have used supercells with two monomers in all our simulations. This allows us also to discriminate between the two different magnetic orders, ferro- and antiferro-magnetic: The ferromagnetic order describes the intrinsic properties of the compound, while the antiferromagnetic order is a prototype for a disordered spin distribution within the polymer, a situation similar to what is expected in the experiments in the absence of an externally applied magnetic field. The ground state electronic structure differs only slightly from the gas phase molecular case, especially for the orbital distribution in both the $l s$ and $h s$ (see Figure S4, Supporting Information). More interesting is the analysis of the spin-resolved electronic transport along the polymer considered here to behave as a quasi one-dimensional wire.

The transport results for polymer $\mathbf{2}$ are summarized in Figure 8. The transmittance spectra for both the $l s$ and $h s$ configurations and both spin channels (Figure 8a,b) display a larger energy gap that corresponds to the states localized in the backbone. In fact, comparing the corresponding density of states (see Figure S4, Supporting Information) we note that the
Co-derived states that populate the energy region around the Fermi energy do not contribute to the quantum transmittance. This is due to the fact that, at variance with the coordinated polymer $\mathbf{1}$, the bridging Co complex is now off the axis of the system. In general, this induces a decrease of the current, as shown in panels $\mathrm{c}$ and $\mathrm{d}$. Here, we can note the following: (i) Most of the current comes from eigenchannels localized in the backbone. This is demonstrated by the almost unpolarized character of the $\alpha$ and $\beta$ transmittance spectra for both magnetic states (see panels a and b) and explains the similar current distribution at high gate voltages for both $l s$ and $h s$ configurations. Similar results are expected for the bare organic polymer without the $\mathrm{Co}(\mathrm{SQ})$ units (2b). (ii) In the $h s$ state, the presence of a number of $\beta$ peaks in the transmittance spectrum within the backbone gap induces a symmetry breaking between $l s$ and $h s$, with the appearance of a diamond shape with maximum current at bias voltages around $0.25 \mathrm{eV}$ but with strong components also at zero gate. In this case, the system can work as a switch also at zero gate voltage. (iii) The analysis of the individual $\alpha$ and $\beta$ components of the current (displayed in Figure 8e) demonstrates that all the current of the $h s$ configuration comes from a single spin state, $\beta$. Note in the insets of panel e the different order of magnitude of the current units (nA vs $\mu \mathrm{A}$ ). This system can thus effectively form the basis for a new type of molecular spintronic device where the VT controls directly spin transport in the active layer through the equivalent of a spin valve effect. Notably, this effect would not be possible in a bare polymer without the Co complex. All the above results refer to the polymer in the ferromagnetic order. The results for the antiferromagnetic case do not differ significantly in any way besides that now the conductances will be the same as the sum of both spin channels (see Figure S6 in the Supporting Information). 
Finally, we want to stress once again that the difference between polymers $\mathbf{1}$ and $\mathbf{2}$ is indeed in the geometry of the bonding of the metal complex with respect to the polymer backbone and thus the coordination of the ligand field. By simply bending polymer 1 at the Co site, the current does not change at all, confirming that the displacement of the Co ion outside the CNpy vertical path significantly reduces the number of states that may contribute to transport in the forbidden region. We can therefore conclude that, as the presence of the lateral dioxolene ligands is responsible for the valence tautomeric character of these compounds, the vertical coupling with the CNpy groups regulates the charge flow through the polymeric axis.

\section{CONCLUSIONS}

Using calculations from first principles and a Landauer approach for charge transport in nanostructures, we predicted that cobalt bis(dioxolene) valence tautomeric complexes exhibit magnetic-state-dependent electronic and spin transport properties and we demonstrated that the VT magnetic bistability translates into a novel form of multifunctional material systems.

Our results also show that the electronic and spin transport properties are strongly structure dependent, an indication of a strong structure-property relation in this class of systems. Overall, valence tautomers represent an exciting new material paradigm for use in spintronic devices (e.g., switches and spin valve sensors). These devices could exploit the predicted intimate interplay between magnetic bistability and metalligand coordination. Moreover, our calculations show that valence tautomerism can be readily integrated into polymeric materials that are fully compatible with state-of-the-art organic electronics processing methodology. This suggests an efficient path to test these material predictions in prototype devices.

\section{ASSOCIATED CONTENT}

\section{S Supporting Information}

Tests on the effects of DFT $+\mathrm{U}$, total energy dependence on $\mathrm{Co}-\mathrm{N}$ distances in polymer $\mathbf{1}$, density of states of polymers 1 and 2, relationship between density of states and quantum transmittance, the main lines of quantum transport method, and $I-V$ characteristics for intermolecular long-range magnetic order. Details of chemical synthesis and characterization of conjugated compounds $\mathbf{2}$ are also reported. Corresponding experimental CIF structures and optimized theoretical geometries are attached in separate files. This material is available free of charge via the Internet at http://pubs.acs.org.

\section{AUTHOR INFORMATION}

\section{Corresponding Author}

*E-mail: arrigo.calzolari@nano.cnr.it; mbn@unt.edu.

\section{Notes}

The authors declare no competing financial interest.

\section{ACKNOWLEDGMENTS}

This work was supported, in part, by NSF-CCI Center for Molecular Spintronics (CHE-0924966). M.B.N. wishes to acknowledge partial support from the Office of Basic Energy Sciences, U.S. Department of Energy at Oak Ridge National Laboratory under contract DE-AC05-00OR22725 with UTBattelle, LLC. Computational resources were provided in part by NCCS-ORNL, HPC-NCSU, and CINECA (project
IscraC SHOCK). We kindly thank M. Rontani for useful discussions and a critical reading of the manuscript.

\section{REFERENCES}

(1) Sanvito, S. Chem. Soc. Rev. 2011, 41, 3336-3355.

(2) Troiani, F.; Affronte, M. Chem. Soc. Rev. 2011, 40, 3119-3129.

(3) Morley, N. A.; Rao, A.; Dhandapani, D.; Gibbs, M. R. J.; Grell, M.; Richardson, T. J. Appl. Phys. 2008, 103, 07F306 (1-3).

(4) Dediu, V.; Murgia, M.; Matacotta, F.; Taliani, C.; Barbanera, S. Solid State Commun. 2002, 122, 181-184.

(5) Dediu, V. A.; Hueso, L. E.; Bergenti, I.; Taliani, C. Nat. Mater. 2009, 8, 707-716.

(6) Xiong, Z. H.; Wu, D.; Vardeny, Z. V.; Shi, J. Nature (London) 2004, 421, 821-824.

(7) Dediu, V.; Hueso, L. E.; Bergenti, I.; Riminucci, A.; Borgatti, F.; Graziosi, P.; Newby, C.; Casoli, F.; De Jong, M. P.; Taliani, C.; Zhan, Y. Phys. Rev. B 2008, 78, 115203 (1-6).

(8) Santos, T. S.; Lee, J. S.; Migdal, P.; Lekshmi, I. C.; Satpati, B.; Moodera, J. S. Phys. Rev. Lett. 2007, 98, 016601 (1-4).

(9) Shim, J. H.; Raman, K. V.; Park, Y. J.; Santos, T. S.; Miao, G. X.; Satpati, B.; Moodera, J. S. Phys. Rev. Lett. 2008, 100, 226603 (1-4).

(10) Barraud, C.; Seneor, P.; Mattana, R.; Fusil, S.; Bouzehouane, K.; Deranlot, C.; Graziosi, P.; Hueso, L.; Bergenti, I.; Dediu, V.; Petroff, F.; Fert, A. Nat. Phys. 2010, 6, 615-620.

(11) Margheriti, L.; Chiappe, D.; Mannini, M.; Car, P.; Sainctavit, P.; Arrio, M.-A.; de Mongeot, F. B.; Cezar, J. C.; Piras, F. M.; Magnani, A.; Otero, E.; Caneschi, A.; Sessoli, R. Adv. Mater. 2010, 22, 5488-5493.

(12) Stepanow, S.; Honolka, J.; Gambardella, P.; Vitali, L.; Abdurakhmanova, N.; Tseng, T.-C.; Rauschenbach, S.; Tait, S. L.; Sessi, V.; Klyatskaya, S.; Ruben, M.; Kern, K. J. Am. Chem. Soc. 2010, 132, 11900-11901.

(13) Majumdar, S.; Laiho, R.; Laukkanen, P.; Vayrynen, I. J.; Majumdar, H. S.; Osterbacka, R. Appl. Phys. Lett. 2006, 89, 122114 $(1-3)$.

(14) Wernsdorfer, W. Int. J. Nanotechnol. 2010, 7, 497-522.

(15) Kyatskaya, S.; Mascaro, J. R. G.; Bogani, L.; Hennrich, F.; Kappes, M.; Wernsdorfer, W.; Ruben, M. J. Am. Chem. Soc. 2009, 131, 15143-15151.

(16) Manriquez, J. M.; Yee, G. T.; McLean, R. S.; Epstein, A. J.; Miller, J. S. Science 1991, 252, 1415-1417.

(17) Li, B.; Kao, C.-Y.; Yoo, J.-W.; Prigodin, V. N.; Epstein, A. J. Adv. Mater. 2011, 23, 3382-3386.

(18) Philipp Gutlich, Y. G.; Goodwin, H. A. Chem. Soc. Rev. 2000, 29, 419-427.

(19) Kahn, O.; Martinez, C. J. Science 1998, 279, 44-48.

(20) Mahfoud, T.; Molnaar, G.; Cobo, S.; Salmon, L.; Thibault, C.; Vieu, C.; Demont, P.; Bousseksou, A. Appl. Phys. Lett. 2011, 99, $053307(1-3)$.

(21) Aravena, D.; Ruiz, E. J. Am. Chem. Soc. 2012, 134, 777-779.

(22) Baadji, N.; Sanvito, S. Phys. Rev. Lett. 2012, 108, 217201 (1-5).

(23) Adam, D. M.; Dei, A.; Rheingold, A. L.; Hendrickson, D. N. J. Am. Chem. Soc. 1993, 115, 8221-8229.

(24) Hendrickson, D. N.; Pierpont, C. G. In Spin Crossover in Transition Metal Compounds II; Gutlich, P., Goodwin, H., Eds.; Topics in Current Chemistry; Springer: Berlin/Heidelberg, 2004; Vol. 234, pp 786-786.

(25) Droghetti, A.; Sanvito, S. Phys. Rev. Lett. 2011, 107, 47201 (14).

(26) Giannozzi, P.; et al. J. Phys.: Condens. Matter 2009, 21, 395502 $(1-19)$.

(27) Perdew, J. P.; Burke, K.; Ernzerhof, M. Phys. Rev. Lett. 1996, 77, 3865-3868.

(28) Vanderbilt, D. Phys. Rev. B 1990, 41, R7892-R7895.

(29) Schmidt, R. D.; Shultz, D. A.; Martin, J. D. Inorg. Chem. 2010, 49, 3162-3168.

(30) Di Valentin, C.; Selloni, A. J. Phys. Chem. Lett. 2011, 2, 22232228.

(31) WanT code, publicly available at www.wannier-transport.org. For details, see ref 32 . 
(32) Calzolari, A.; Marzari, N.; Souza, I.; Buongiorno Nardelli, M. Phys. Rev. B 2004, 69, 035108 (1-10).

(33) Landauer, R. Philos. Mag. 1970, 21, 863-867.

(34) Datta, S. Electronic transport in mesoscopic systems; Cambridge University Press: Cambridge, U.K., 1995.

(35) Marzari, N.; Vanderbilt, D. Phys. Rev. B 1997, 56, 12847-12865. (36) Ferretti, A.; Calzolari, A.; Bonferroni, B.; Di Felice, R. J. Phys.: Condens. Matter 2007, 19, 036215 (1-16).

(37) Calzolari, A.; Ferretti, A.; Buongiorno Nardelli, M. Nanotechnology 2007, 18, 424013 (1-8).

(38) Ferretti, A.; Calzolari, A.; Di Felice, R; Manghi, F.; Caldas, M. J.; Buongiorno Nardelli, M.; Molinari, E. Phys. Rev. Lett. 2005, 94, $116802(1-4)$.

(39) O'Sullivan, T. J.; Djukic, B.; Dube, P. A.; Lemaire, M. T. Chem. Commun. 2009, 1903-1905.

(40) Welte, L.; Calzolari, A.; Di Felice, R.; Zamora, F.; GomezHerrero, J. Nat. Nanotechnol. 2010, 5, 110-115.

(41) Jung, O.; Pierpont, C. J. Am. Chem. Soc. 1994, 116, 2229-2230. (42) Brandbyge, M.; Sørensen, M. R.; Jacobsen, K. W. Phys. Rev. B 1997, 56, 14956-14959. 